\title{
Resonant scattering as a cause for the registration of scattering layers in the thermosphere and imaginary aerosol formations in the middle atmosphere
}

\author{
Vasily Bychkov ${ }^{1}$, Andrey Perezhogin and Ilya Seredkin \\ Institute of Cosmophysical Research and Radio Wave Propagation of the Far Eastern Branch of \\ Russian Academy of Sciences, Paratunka, Russia
}

\begin{abstract}
The results of lidar and ionospheric observations from August to November 2017 are discussed. Resonance scattering was detected at wavelengths of 532.08 and $561.106 \mathrm{~nm}$ in the altitude range of 200-400 km and in the middle atmosphere. A possible mechanism for the formation of a resonant scattering signal on excited ions of the main gas components of the atmosphere is presented. The possibility of estimating the spectra of precipitated electrons is shown.

It is shown that the complete profile of the backscattering signal can be restored in the region of 10-25 km using an additional channel for recording the attenuated signal separated from the main signal. Thus, it becomes possible to estimate the energies of electron flows in the entire region from the lower thermosphere to the stratosphere.
\end{abstract}

\section{Introduction}

The results of a study of resonance scattering in the upper atmosphere according to lidar and ionospheric data for September 5 and 23, 2017 are presented in [1] - [2]. It was shown that the observed light scattering in the region of the lower thermosphere and in the mesosphere can be explained by resonance scattering by excited ions of atomic nitrogen and oxygen. Resonance scattering at wavelengths of 532 and $561 \mathrm{~nm}$ can be recorded over the entire range of heights, starting from the lower boundary of the stratosphere. This is due to the fact that the time between collisions of excited ions with neutral components of the atmosphere exceeds the lifetime of these states,. The Doppler broadening of the lines at the temperature of $200 \circ \mathrm{K}$ (mesopause) is $0.002 \mathrm{~nm}$ and is still quite sufficient for the formation of resonance scattering conditions.

The results of lidar studies of the middle atmosphere of Kamchatka conducted in 20072012 are presented in [3]. According to these results, aerosol scattering at the altitudes of the stratosphere above Kamchatka can appear in the cold season, from November to March, and is absent from May to October. Weak aerosol formations at altitudes near $70 \mathrm{~km}$ were observed in all seasons. In the present work a more accurate substantiation of the mechanism of the formation of the backscattering signal on excited ions of atomic nitrogen and oxygen is carried out. The optical scheme of the lidar for obtaining the return signal in

1 Corresponding author: vasily.v.bychkov@gmail.com 
the entire region of the stratosphere is discussed. For convenience of presentation and perception of the text, the used equipment and the table of dipole transitions are reproduced.

\section{Hardware complex}

In the observations of 2017 a two-frequency lidar with Nd:YAG laser was used to generate the radiation at the wavelength of $532 \mathrm{~nm}$ and a dye laser to generate the radiation at the wavelength of $561.1 \mathrm{~nm}$ at the frequency of $10 \mathrm{~Hz}$ The main parameters of the lidar used in the experiments are shown in Table 1.

Table 1. Equipment.

\begin{tabular}{|l|l|l|}
\hline \multicolumn{1}{|c|}{ Transmitter 1 } & \multicolumn{1}{|c|}{ Transmitter 2 } & \multicolumn{1}{c|}{ Receiver } \\
\hline Laser Nd:YAG Brilliant-B & Dye Laser TDL-90 & Telescope Diameter 60 cm \\
Pulse Energy 400 mJ & Pump Laser YG-982E & PMT Hamamatsu H8259-01 \\
Wavelength 532.08 nm & Pulse Energy 100 mJ & Photon Counters M8784-01, \\
Linewidth 0.04 nm & Wavelength 561.106 nm & Spatial resolution 1.5 km \\
\hline
\end{tabular}

Figure 1 shows the optical scheme of the lidar. The clock pulse SP is generated by the pump laser and is applied to the Nd:YAG laser of the first channel, the TDL laser, the photoelectron multiplier, the photon counters, and the control unit. The main function of the CU (control unit) is to form two locking pulses having a voltage of $5 \mathrm{~V}$ and a preset duration and to apply them simultaneously with the clock pulse to both photoelectron multipliers to exclude PMT illumination from near zone signals.

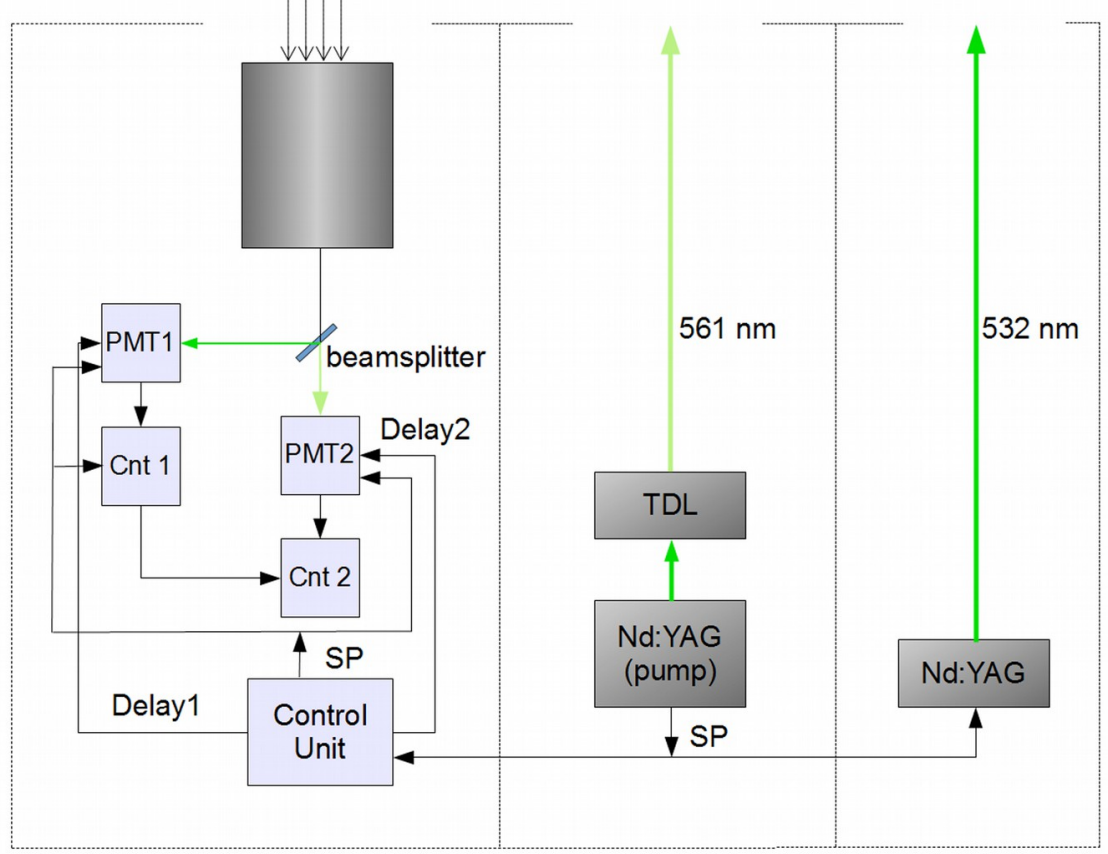

Fig. 1. Optical scheme of the lidar. Here SP is the clock pulse, Cnt is the photon counter, PMT is the photoelectron multiplier tube, and CU is the control unit. 
The scheme of the studied dipole transitions is given in the Table 2 [4]. Here OII - ion $\mathrm{O}^{+}$, NII - ion $\mathrm{N}^{+}, \mathrm{NIII}-$ ion $\mathrm{N}^{++}$.

Table 2. Dipole transitions of excited ions of oxygen and nitrogen atoms falling within the radiation bands of lasers

\begin{tabular}{|l|c|c|l|l|l|l|l|l|l|}
\hline & $\begin{array}{c}\text { Compo- } \\
\text { nent }\end{array}$ & $\begin{array}{c}\text { Wavelength } \\
\text { Air }(\mathrm{nm})\end{array}$ & $\mathrm{A}_{\mathrm{ki}}\left(\mathrm{s}^{-1}\right)$ & Lower Level & Term & $\mathrm{J}$ & Upper Level & Term & $\mathrm{J}$ \\
\hline 1 & O II & 561.1072 & $2.14 \mathrm{e}+06$ & $2 \mathrm{~s}^{2} 2 p^{2}\left({ }^{1} \mathrm{~S}\right) 3 \mathrm{~s}$ & ${ }^{2} \mathrm{~S}$ & $1 / 2$ & $2 \mathrm{~s}^{2} 2 p^{2}\left({ }^{3} \mathrm{P}\right) 4 p$ & ${ }^{2} \mathrm{P}^{\circ}$ & $1 / 2$ \\
\hline 2 & NIII & 532.0870 & $5.68 \mathrm{e}+07$ & $2 \mathrm{~s} 2 \mathrm{p}\left({ }^{3} \mathrm{P}^{\circ}\right) 3 \mathrm{p}$ & ${ }^{2} \mathrm{D}$ & $5 / 2$ & $\left.2 \mathrm{~s} 2 \mathrm{p}^{3} \mathrm{P}^{\circ}\right) 3 \mathrm{~d}$ & ${ }^{2} \mathrm{~F}^{\circ}$ & $7 / 2$ \\
\hline 3 & NII & 532.0958 & $2.52 \mathrm{e}+07$ & $2 \mathrm{~s} 2 \mathrm{p}^{2}\left({ }^{4} \mathrm{P}\right) 3 \mathrm{p}$ & ${ }^{5} \mathrm{P}^{\circ}$ & 1 & $2 \mathrm{~s} 2 \mathrm{p}^{2}\left({ }^{4} \mathrm{P}\right) 3 \mathrm{~d}$ & ${ }^{5} \mathrm{P}$ & 2 \\
\hline
\end{tabular}

The lines represented in Table 2 were chosen on the basis of the laser radiation band widths and ion line Doppler broadening at the ionospheric heights of $\sim 0.04 \mathrm{~nm}$ for the temperature of $800^{\circ} \mathrm{K}$.

Figure 2 presents the location of the lines of excited ions in the spectra of lasers.

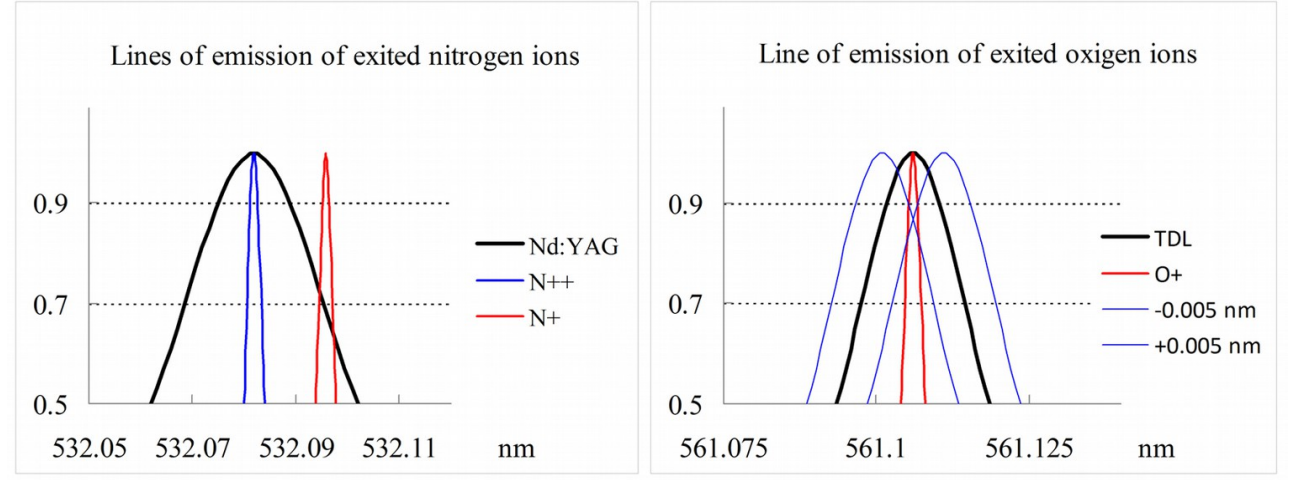

Fig. 2. Emission lines of excited oxygen and nitrogen ions in the emission spectra of Nd: YAG and TDL lasers.

The blue lines in the right part of the figure show the shift of the laser emission spectrum relative to the oxygen ion emission line, due to a possible error in the TDL laser calibration. It is assumed that the laser wavelength is set to an accuracy of $0.01 \mathrm{~nm}$.

The line of the twice-ionized nitrogen ion falls into the center of the laser emission band, but the content of such atoms is small. The main contribution to the lidar signal is made by $\mathrm{N}^{+}$ions.

\section{Experimental data}

The spatial-temporal distribution of the return signal received on August 3, 2017 in Fig. 1 is presented. It uses lidar data with a 15-minute accumulation and a spatial resolution of 1.5 $\mathrm{km}$. The background signal was subtracted. The presented data are very similar to the results obtained on September 5, given in [1] and have the same main features:

- the altitude of the lidar return signal maximum did not correlate with the location of the F2 layer maximum. The lidar signal was maximal at altitudes $280-290 \mathrm{~km}$. This approximately corresponded to the altitude of the F2 layer maximum in the daytime and in the evening. At night, the maximum altitude increased, since the recombination rate in the lower layers is higher, whereas the ionization sources are absent. 
- Enhanced light scattering forming the second local maximum (Fig. 3) was observed for all total lidar signals at a wavelength of $561 \mathrm{~nm}$ at altitudes $300-400 \mathrm{~km}$. In all the cases, the signal at a wavelength of $532 \mathrm{~nm}$ decreased monotonically from its maximal value with increasing altitude.

- the signal levels at two wavelengths were approximately identical. The values of the total signal at a wavelength of $532 \mathrm{~nm}$ were usually by $20-30 \%$ higher. It was expected that the lidar signal at a wavelength of $561 \mathrm{~nm}$ would be several times higher than the signal at a wavelength of $532 \mathrm{~nm}$ since the content of $\mathrm{O}^{+}$ions at altitudes $150-400 \mathrm{~km}$ is approximately two orders of magnitude higher than that of $\mathrm{N}^{+}$ions [5].
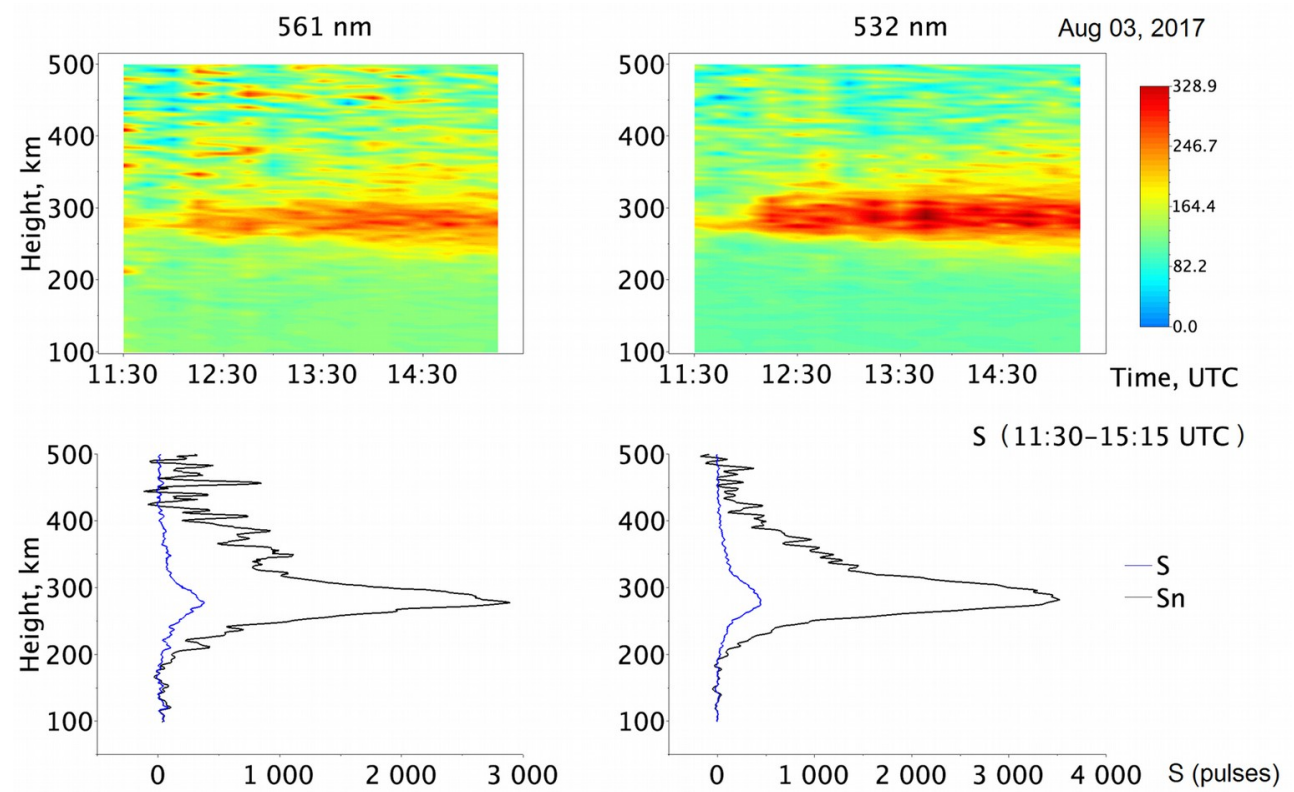

Fig. 3. Lidar «signal-background» in the region of $100-500 \mathrm{~km}$, total signal $\mathrm{S}$ and normalized signalSn during the lidar observations on August 3, 2017

\section{Discussion}

\subsection{Lower thermosphere}

The first two features of the lidar signal are explained by the conclusions made in [1] that when ionization sources appear, excited ions of nitrogen and oxygen atoms appear both during ionization of nitrogen and oxygen molecules, and upon excitation of existing ions. The first process forms the main signal maximum of the region of $290 \mathrm{~km}$. According to [5], $60 \%$ of oxygen ions are formed in excited states. The second causes an increase in the signal level in the region of the maximum of the F2 layer only on the signal profile with a wavelength of $561 \mathrm{~nm}$. The data presented confirm this.

The exceed of the signal level at a wavelength of $532 \mathrm{~nm}$ over the signal obtained at a wavelength of 561 is explained by the scattering formation mechanism. The signal estimation method proposed in [1] requires refinement.

The signal should be proportional to the pulse energy and the content of excited ions.Not all photons of the probe pulse will participate in the formation of the scattering signal, but only those whose energy falls into the emission line. Figure 2 illustrates this clearly. 
Taking into account the Doppler line broadening equal to $\sim 0.004 \mathrm{~nm}$ for both ions and the data of Table 1 and Figure 2, it will be $\sim 10 \%$ for radiation at a wavelength of $532 \mathrm{~nm}$ and $\sim 15 \%$ for radiation at a wavelength of $561 \mathrm{~nm}$. The "useful" pulse energy will be 40 and 15 $\mathrm{mJ}$ for radiation at wavelengths of 532 and $561 \mathrm{~nm}$, respectively.

To evaluate the content of excited tones, it is necessary to take into account that the lower level states of Table 1 are also excited. We will consider the content of excited ions in an thin compared to the value of the strobes $(150$ and $300 \mathrm{~m})$ layer. At the moment of arrival of the laser pulse, the content of excited ions in it is proportional to the lifetime of the state $\tau$ multiplied by the rate of formation of ions in this state. The rate of formation of oxygen ions is three times higher than that of nitrogen ions [5]. The interaction of the laser pulse with the selected layer continues for a time $\mathrm{T}$, where $\mathrm{T}$ is the pulse duration.In [1], values of $\tau$ equal to $1.06,1.42$, and $12.82 \mathrm{~ns}$ for $\mathrm{O}^{+}, \mathrm{N}^{+}$, and $\mathrm{N}^{+}$, respectively, were calculated. The pulse durations (Table 1) are 10 and $5 \mathrm{~ns}$ for 561 and $532 \mathrm{~nm}$.

Substitution of all obtained estimated values gives a signal ratio of 0.7 . It must also be taken into account that the cross section for resonance scattering is proportional to the square of the wavelength, and the number of photons in a pulse is proportional to the length of the wave. The wavelength ratio in $(561 / 532)^{3}=1.17$ and, accordingly, the signal ratio should be equal to 0.82 .

The total signal should be proportional to the ion formation rate multiplied by the interaction time $\left(T_{\text {pulse }}+\tau\right)$ and multiplied by "useful" energy of $E_{\text {pulse. }}$ The results obtained are in agreement with these estimation

The spectrum of precipitated electrons can be estimated using the data of [5-8] according to the method described in [1]. In a specific case, on August 3, 2017, it can be defined as monoenergetic with an energy of $\sim 300 \mathrm{eV}$.

\subsection{Middle atmosphere}

In the lidar studies of the middle atmosphere, the scattering ratio parameter $R=(\beta a+\beta m) /$ $\beta \mathrm{m}=1+\beta \mathrm{a} / \beta \mathrm{m}$ was used, where $\beta \mathrm{a}, \beta \mathrm{m}$ are the aerosol and molecular scattering coefficients. The values of $\mathrm{R}$ above unity indicate the appearance of aerosol formations. In the ordinary state of the mesosphere and stratodphere there are no conditions for water condensation and aerosol formation.

When ionization sources appear, the conditions for the appearance of resonant scattering in the middle atmosphere are preserved. For a temperature of $800^{\circ} \mathrm{K}$ (layer F2), it is 0.004 $\mathrm{nm}$. The Doppler broadening of the lines at the temperature of $200^{\circ} \mathrm{K}$ (mesopause) is $0.002 \mathrm{~nm}$ and is still quite sufficient for the formation of resonance scattering conditions.

The electron-neutral collision frequency can be calculated from: $v[\mathrm{~N}]=0.81 \cdot 10^{-10}$ $(\mathrm{T} / \mathrm{M}) 1 / 2 \mathrm{~N}\left(\mathrm{c}^{-1}\right)$, where $\mathrm{T}$ is the temperature, $\mathrm{M}$ is the molecular weight, and $\mathrm{N}$ is the concentration of atmospheric particles [9]. Substituting the value of $\mathrm{N}$, for an altitude of $10 \mathrm{~km}$ equal $8 \cdot 10^{18} \mathrm{~cm}^{-3}$, we get $\vee 4 \cdot 10^{9} \mathrm{c}^{-1}$, or $4 \mathrm{GHz}$. If we take into account that the frequency of ion-neutral collisions is at least two or three orders of magnitude lower than that of electrons, we can conclude that for excited ions with a radiation lifetime $10^{-8}-10^{-9} \mathrm{c}$ the increase in the number of collisions at altitudes of $10 \mathrm{~km}$ and above does not play any noticeable role. 2017

Figure 4 shows the scattering coefficient $\mathrm{R}$ profiles plotted from data for September 5 , 

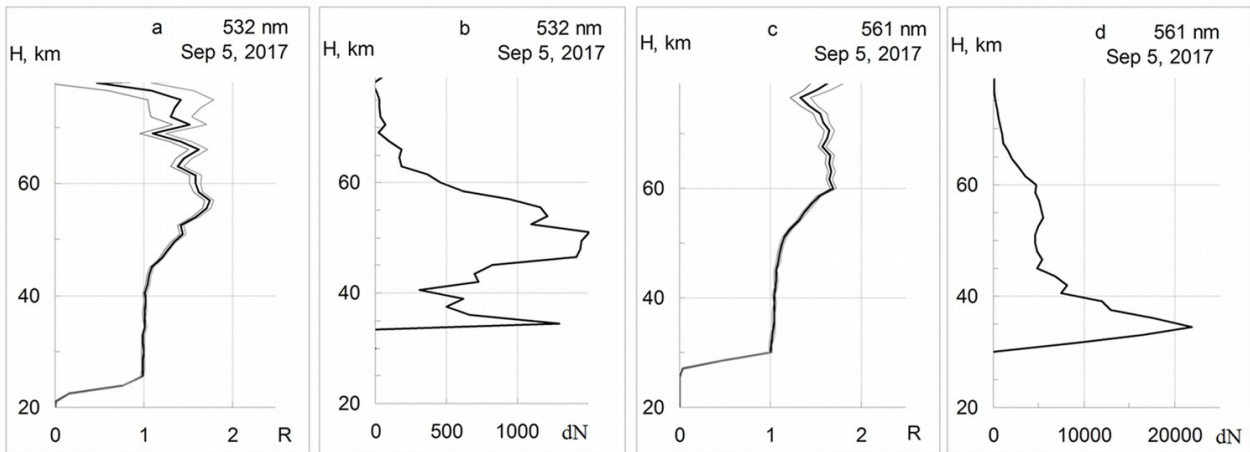

Fig. 4. The scattering ratio profiles $\mathrm{R}$ obtained on September 5, 2017 (a, c) and dN - the total lidar signal net of "the molecular scattering signal and the background signal" (b, d).

The value of $\mathrm{dN}$ is determined as: $\left.\mathrm{S}=\left(\mathrm{N}-\mathrm{N}_{\mathrm{f}}\right) \cdot(\mathrm{R}-1) / \mathrm{R}\right)$, where $\mathrm{N}-$ is the total lidar signal, $\mathrm{N}_{\mathrm{f}}$ - is the total background signal, $\mathrm{R}$ - scattering ratio. Thus, $\mathrm{dN}$ is an additional signal due to the presence of an aerosol or resonant scattering.

In [1], the data of Fig. $4 \mathrm{~b}$ were analyzed. It was shown that the additional signal $\mathrm{dN}$ has a maximum in the region of 47-51 km and is located in the region of 40-65 km. At these altitudes, ionization is produced by electrons with an energy of $600 \mathrm{keV}$. An increase in the additional signal from a height of 40 to $35 \mathrm{~km}$ allows us to conclude that there were observed precipitations of electrons with energies greater than $2.8 \mathrm{MeV}$.

Fig. 5 shows similar data for 03.08.2017.

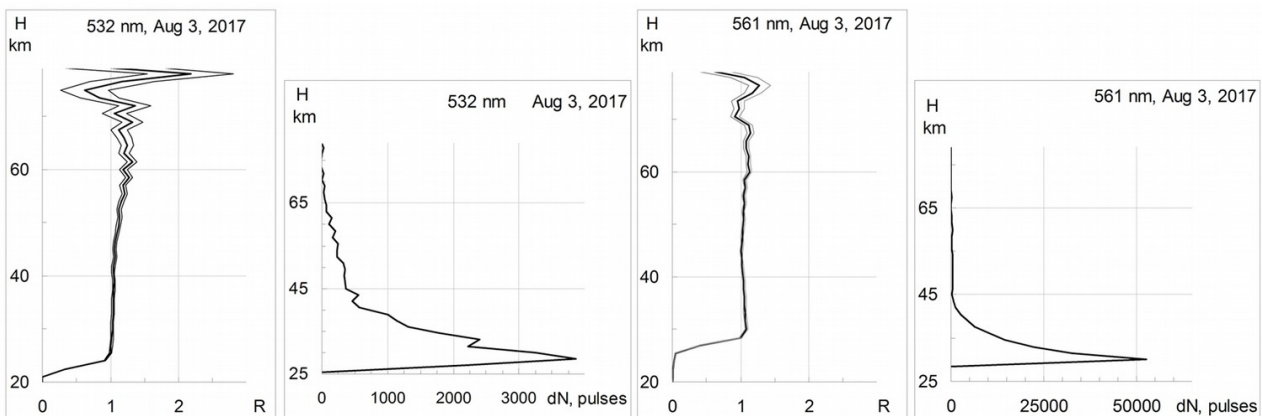

Fig. 5. The scattering ratio profiles $\mathrm{R}$ obtained on August 3, 2017 and $\mathrm{dN}$ - the total lidar signal net of "the molecular scattering signal and the background signal".

In general, such scattering ratio profiles R are typical for Kamchatka in the "warm" season" from April to October. Slight increases in the ratio on both profiles in the region of 50$70 \mathrm{~km}$ can be caused by weak precipitations of electrons with energies in the range 100-300 $\mathrm{keV}$. But they can also be explained by the difference in temperature values at these heights from the model NRLMSIS-00 that values used in the calculation [10]. The values of the molecular scattering coefficient and backscattering signal at these heights are small and the error increases.

Noteworthy is the presence in both receiving channels on both days of an additional signal dN, which, starting from a height of $\sim 40 \mathrm{~km}$, increases monotonically when height decreases. This additional signal does not significantly affect the shape of the scattering ratio profile, since it is less than $1 \%$ of the molecular scattering signal from these heights. 
In addition, in both figures in this region, the signal at a wavelength of $561 \mathrm{~nm}$ is more than an order of magnitude greater than the signal at a wavelength of $532 \mathrm{~nm}$. If this signal is caused by ionization sources, then the difference in the magnitude of the signals can be explained by the presence of ozone in this region. But it is still difficult to draw any conclusions. At the bottom of the profile, the signal breaks off, because this altitude region is not recorded by lidar. What is caused by the need to use electronic PMT locking to avoid overloading it.

The solution to the problem may be the modernization of the lidar system. It is necessary to install an additional dividing plate of ordinary optical glass in each of the receiving channels of the lidar. Between the PMT and the beam splitter in Figure 1.The reflected few percent of the main signal can then be sent to other PMT, on which the delay pulse can be reduced. This modernization will not have a significant impact on the main signal and will enable the stratosphere to be included in the range of probed heights.

\section{Conclusion}

New lidar data confirming the possibility of detecting resonance scattering on gas components are presented.

A possible mechanism for the formation of a lidar signal of resonance scattering by ex cited ions of atomic nitrogen and oxygen is proposed. The total lidar signal should be proportional to the ion formation rate multiplied by the interaction time (Tpulse $+\tau$ ) and multi plied by the pulse energy falling into the spectrum of Doppler broadening of the lines.

The possibility of the formation of imaginary aerosol layers in the mesosphere and stratosphere is substantiated. The Doppler broadening of the lines in middle atmosphere is sufficient for the formation of resonance scattering conditions. An increase in the number of ion-neutral collisions at altitudes of $10 \mathrm{~km}$ and above does not play a noticeable role.

\section{Acknowledgments}

The work was carried out by the means of the Common Use Center "North-Eastern Heliogeophysical Center"CKP_558279, USU 351757 with partial financial support from the RFBR grant No. 19-05-00543A.

\section{References}

1. V.V. Bychkov, A.S. Perezhogin, I.N. Seredkin, E3S Web Conf, 62 (2018)

2. V.V. Bychkov, A.S. Perezhogin, I.N. Seredkin, B.M. Shevtsov, SPIE Proc., 10833A4 (2018)

3. V.V. Bychkov, A.S. Perezhogin, I.N. Seredkin et al., Atmos Ocean Opt, 25: 228 (2012)

4. A. Kramida, Yu. Ralchenko, J. Reader and NIST ASD TEAM, NIST Atomic Spectra

Database (ver. 5.5.2), Gaithersburg, MD (2018)

5. P. G. Richards, JGR, 116, A08307, (2011)

6. M.G. Deminov, Plasma heliogeophysics, (Fizmatlit, Moscow, 2008)

7. M.N. Rees, P. C. Benedict, JGR, 75 (1970)

8. Omholt, A., Stoffregen, W., Derblum, H.: 1962, J. Atmosph. Terr. Phys, 24, 203

9. N.N. Shefov, A.I. Semenov, V.Yu. Chomich, Radiation of the upper atmosphere an indicator of its structure and dynamics, (GEOS, Moscow, 2006)

10. M. Picone, A.E. Hedin, D. Drob, NRLMSISE-00 Model 2001, https://ccmc.gsfc.nasa.gov/modelweb/atmos/nrlmsise00.html 